\title{
Analytical description of hadronic integral spectra of cosmic-ray superfamilies with Feynman scaling breaking
}

\author{
J. Bellandi, ${ }^{1}$ C.G.S. Costa, ${ }^{2}$ R.J.M. Covolan, ${ }^{1}$ C. Dobrigkeit, ${ }^{1}$ L.M. Mundim, ${ }^{1}$ and C. Salles ${ }^{1}$ \\ ${ }^{1}$ Instituto de Física Gleb Wataghin, Universidade Estadual de Campinas, 13083-970, Campinas, São Paulo, Brasil \\ ${ }^{2}$ Instituto de Física Teórica, Universidade Estadual Paulista, 0145-900, São Paulo, São Paulo, Brasil
}

(Received 6 April 1994)

\begin{abstract}
We describe charged hadronic energy spectra of the cosmic-ray superfamilies named Ursa Maior and P3'-C1-B90, detected by the Chacaltaya and Pamir Emulsion Chamber Collaborations, respectively, using analytical solutions for the diffusion equations. We obtain a very good description of these events incorporating Feynman scaling breaking in the multiparticle production model of our cascading formalism. Centauro exotic events are also investigated.
\end{abstract}

PACS number(s): $13.85 . T p, 96.40 . D e$

\section{INTRODUCTION}

Superfamilies of cosmic radiation [1] have attracted the attention of many emulsion-chamber experimental collaborations $[2,3]$, because among them the most energetic shower events produced in the atmosphere are found [4], as well as the challenging and not yet well understood exotic events, such as Centauro events $[1,5]$.

In the extremely high energy region (with energies in the laboratory system above $1000 \mathrm{TeV}$ ), the cosmic-ray families detected in emulsion chambers are frequently accompanied by a "halo," i.e., a uniform blackening of the $x$-ray film around the central core. The first observation of a huge halo event was "Andromeda," detected in 1969 at Chacaltaya chamber No. 14 [6], whose total visible (hadronic and electromagnetic) energy was $\sum E_{\text {vis }}^{\text {halo }} \approx 21000 \mathrm{TeV}(\sqrt{s} \approx 6.5 \mathrm{TeV}$, in the centerof-mass system). This is still far beyond the energy region of the most powerful particle accelerators now in operation. However, there are superfamilies with a total visible energy (of the off-halo part) at the frontiers now reached by colliders, such as the family "Ursa Maior," with $\sum E_{\text {vis }}=2174 \mathrm{TeV}(\sqrt{s} \approx 2 \mathrm{TeV})$ detected by the Brazil-Japan Collaboration [7] and the event "P3'-C1B90," with $\sum E_{\text {vis }}=1042 \mathrm{TeV}(\sqrt{s} \approx 1.4 \mathrm{TeV})$ detected by the Pamir Collaboration [3].

These facts led to renewed interest in analyzing such cosmic-ray events, using accelerator-based parametrizations, with no need for extrapolations. One of these parametrizations is the multiparticle production spectrum proposed by Bellandi et al. [8], which was recently reviewed [9] to fit charged-particle pseudorapidity density distributions collected by the UA5 [10] and UA7 [11] Collaborations at the CERN collider, and is found to be consistent with collider detector at Fermilab (CDF) measurements at the Tevatron collider [12], up to $\sqrt{s}=$ $1.8 \mathrm{TeV}$. The pseudorapidity density parametrization reflects the fact that the Feynman scaling law is violated at accelerator energies above $\sqrt{s}=60 \mathrm{GeV}$ [13], in both central and fragmentation regions [9].

Another crucial question of concern here is the inelasticity problem. It is discussed in Ref. [9] that the charged multiparticle distributions referred to above imply a smooth decrease of inelasticity with energy. It has been shown that such a behavior of multiparticle production is not compatible with some cosmic-ray data, such as the altitude dependence of the intensity of electromagnetic component [9], the energy spectrum of nucleonic component [14], and recent data from Fly's Eye experiment [15], all of them concerning showers generated by a primary cosmic ray at the top of the atmosphere. However, that question still remains unsolved for the case of single-particle generated events, such as superfamilies. Partially, this is because there are several mathematical difficulties in solving the diffusion equations for family shower development, in order to perform the proper analysis. Generally, solutions of those diffusion equations are written as integrals in the complex plane, requiring approximative methods of calculation, such as the saddlepoint method (e.g., Ref. [16]).

We present in this paper a formalism, based on the Feynman procedure of ordered exponential operators [17], which enables us to solve analytically the diffusion equations for the hadronic cascade induced by one single nucleon in the atmosphere, writing the final solution in the energy configuration space (Sec. II). With this solution, we examine in Sec. III whether or not it is possible to describe the hadronic integral spectra of the two cosmic-ray families mentioned above, "Ursa Maior" and "P3'-C1-B90." First we adopt the Feynman scaling law, just to see explicitly the problems that arise, and then we use the violation of scaling as stated by the multiparticle production spectrum of Ref. [9]. In the latter case, it is necessary to choose proper distributions for the nucleonic and pionic elasticities. The formalism used to describe the two previous families is also applied to investigate the event "Centauro I." Finally, in Sec. IV, we present our discussion and concluding remarks.

\section{ANALYTICAL SOLUTION OF SUPERFAMILY DIFFUSION EQUATIONS}

In order to analyze the charged hadronic integral spectrum for those family events by means of the analyti- 
cal solutions of the diffusion equations we assume here that (i) we have only nucleons and pions in the hadronic cascade; (ii) the leading particle model is adopted, and (iii) there is a consistency condition between the leading charged nucleon and the charged multiparticle production distributions, in the sense that the mean charged nucleon elasticity $\left\langle\sigma_{N}\right\rangle$ is related to the average inelasticity $\langle K\rangle$ for the charged multiparticle production by
$\left\langle\sigma_{N}\right\rangle+\langle K\rangle=1$. (iv) We neglect the production of pions by pions and the pionic decay.

Let $F_{i}\left(E, E_{0}, t, t_{0}\right) d E d t$ (with $i=N, \pi$ ) be the nucleonic or pionic flux with energy between $E$ and $(E+d E)$, at the detection level between $t$ and $(t+d t)$, for the cascade generated at atmospheric depth $t_{0}$ by a single nucleon (here supposed to be a proton) with definite energy $E_{0}$. The diffusion equations are

$$
\begin{aligned}
\frac{d F_{N}}{d t}\left(E, E_{0}, t, t_{0}\right)=- & \frac{1}{\lambda_{N}} F_{N}\left(E, E_{0}, t, t_{0}\right)+\frac{1}{\lambda_{N}} \int_{0}^{1} f\left(\sigma_{N}\right) F_{N}\left(E / \sigma_{N}, E_{0}, t, t_{0}\right) \frac{d \sigma_{N}}{\sigma_{N}}, \\
\frac{d F_{\pi}}{d t}\left(E, E_{0}, t, t_{0}\right)= & -\frac{1}{\lambda_{\pi}} F_{\pi}\left(E, E_{0}, t, t_{0}\right)+\frac{1}{\lambda_{\pi}} \int_{0}^{1} f\left(\sigma_{\pi}\right) F_{\pi}\left(E / \sigma_{\pi}, E_{0}, t, t_{0}\right) \frac{d \sigma_{\pi}}{\sigma_{\pi}} \\
& +\frac{1}{\lambda_{N}} \int_{E}^{E_{0}} g_{N}\left(E, E^{\prime}\right) F_{N}\left(E^{\prime}, E_{0}, t, t_{0}\right) d E^{\prime}
\end{aligned}
$$

where $\lambda_{i}$ are the average interaction mean free paths, here assumed $\lambda_{N}=80 \mathrm{~g} / \mathrm{cm}^{2}$ and $\lambda_{\pi}=120 \mathrm{~g} / \mathrm{cm}^{2} ; f\left(\sigma_{i}\right)$ are the elasticity fluctuation functions; and $g_{N}\left(E, E_{0}\right)$ is the multiparticle production function of charged pions by nucleons.

The first (second) term on the right-hand side (RHS) of both Eqs. (1) and (2) corresponds to the decrease (increase) of the flux due to the diffusion of leading particles through the atmosphere; the third term on the RHS of Eq. (2) is related to the multiparticle production of pions by nucleons.

The diffusion equations (1) and (2) are solved under the boundary conditions

$$
\begin{aligned}
F_{N}\left(E, E_{0}, t\right. & \left.=t_{0}\right)=\delta\left(E-E_{0}\right), \\
F_{\pi}\left(E, E_{0}, t=t_{0}\right) & =0 .
\end{aligned}
$$

Following condition (iii), we choose for the charged nucleonic and pionic elasticity fluctuations the normalized distributions

$$
f\left(\sigma_{i}\right)=\left(1+\beta_{i}\right) \sigma_{i}^{\beta_{i}} \quad(i=N, \pi),
$$

so that the average elasticities are given by

$$
\left\langle\sigma_{i}\right\rangle=\frac{1+\beta_{i}}{2+\beta_{i}} \quad(i=N, \pi) .
$$

In the special case $\beta_{N}=0$ the elasticity distribution is uniform, so the mean elasticity is equal to 0.5 , and therefore condition (iii) is not necessarily true, depending on the value of $\langle K\rangle$. To make sure condition (iii) is satisfied, we get for the parameter $\beta_{N}$ the expression

$$
\beta_{N}=\frac{1}{\left\langle K\left(E_{0}\right)\right\rangle}-2,
$$

where $\langle K(E)\rangle$ is the mean inelasticity for the charged multiparticle production.

The solutions of Eqs. (1) and (2) can be obtained without any approximations, using a Feynman-like procedure of ordered exponential operators [17]. This is done combining the method already applied to the solution of the hadronic diffusion equations, subjected to a power-law energy spectrum as boundary condition [18], with that applied to solve the nucleonic cascade, induced by one single nucleon [19].

We first introduce the operators $\hat{\sigma}_{i}(i=N, \pi)$ and $\hat{\Sigma}_{N}$, defined by

$$
\begin{aligned}
\hat{\sigma}_{i} F_{i}\left(E, E_{0}\right) & =\int_{0}^{1} f\left(\sigma_{i}\right) F_{i}\left(E / \sigma_{i}, E_{0}\right) \frac{d \sigma_{i}}{\sigma_{i}}, \\
\hat{\Sigma}_{N} F_{N}\left(E, E_{0}\right) & =\int_{E}^{E_{0}} g\left(E, E^{\prime}\right) F_{N}\left(E^{\prime}, E_{0}\right) d E^{\prime},
\end{aligned}
$$

so that the diffusion equations (1) and (2) are written in the form

$$
\begin{aligned}
\frac{\partial F_{N}}{\partial t}\left(E, E_{0}, t, t_{0}\right)= & -\frac{1}{\lambda_{N}}\left[1-\hat{\sigma}_{N}\right] F_{N}\left(E, E_{0}, t, t_{0}\right), \\
\frac{\partial F_{\pi}}{\partial t}\left(E, E_{0}, t, t_{0}\right)= & -\frac{1}{\lambda_{\pi}}\left[1-\hat{\sigma}_{\pi}\right] F_{\pi}\left(E, E_{0}, t, t_{0}\right) \\
& +\frac{1}{\lambda_{N}} \hat{\Sigma}_{N} F_{N}\left(E, E_{0}, t, t_{0}\right),
\end{aligned}
$$

which have the formal solutions

$$
\begin{aligned}
F_{N}\left(E, E_{0}, t, t_{0}\right)= & \exp \left\{-\frac{\left(t-t_{0}\right)}{\lambda_{N}}\left[1-\hat{\sigma}_{N}\right]\right\} \\
& \times F_{N}\left(E, E_{0}, t=t_{0}\right) \\
F_{\pi}\left(E, E_{0}, t, t_{0}\right)= & \int_{t_{0}}^{t} d z \exp \left\{-\frac{(t-z)}{\lambda_{\pi}}\left[1-\hat{\sigma}_{\pi}\right]\right\} \\
& \times \frac{1}{\lambda_{N}} \hat{\Sigma}_{N} F_{N}\left(E, E_{0}, z, t_{0}\right) .
\end{aligned}
$$

The explicit solution for the nucleonic component was already calculated in Ref. [19] and, with little modifications to include the more general elasticity distribution, Eq. (5), we have 


$$
F_{N}\left(E, E_{0}, t, t_{0}\right)=e^{-T_{N}}\left[\delta\left(E-E_{0}\right)+\frac{T_{N}}{E}\left(\frac{E}{E_{0}}\right)^{1+\beta_{N}}\left(1+\beta_{N}\right) \frac{2 I_{1}\left(u_{0}\right)}{u_{0}}\right]
$$

where $I_{1}(u)$ is the modified Bessel function of first order and

$$
\begin{aligned}
T_{N} & =\frac{t-t_{0}}{\lambda_{N}} \\
u_{0} & =2\left[\left(1+\beta_{N}\right) T_{N} \ln \left(E_{0} / E\right)\right]^{1 / 2} .
\end{aligned}
$$

For the pionic component we note that the formal solution given by Eq. (13) can be written as

$$
\begin{aligned}
F_{\pi}\left(E, E_{0}, t, t_{0}\right)= & \int_{t_{0}}^{t} \frac{d z}{\lambda_{N}} \int_{E}^{E_{0}} d E^{\prime \prime}\left[\exp \left\{-\frac{(t-z)}{\lambda_{\pi}}\left(1-\hat{\sigma}_{\pi}\right)\right\} \delta\left(E-E^{\prime \prime}\right)\right] \\
& \times \int_{E^{\prime \prime}}^{E_{0}} d E^{\prime} \hat{\Sigma}_{N} \delta\left(E^{\prime \prime}-E^{\prime}\right) F_{N}\left(E^{\prime}, E_{0}, z, t_{0}\right) .
\end{aligned}
$$

We know, by Eq. (14), the solution for $F_{N}\left(E^{\prime}, E_{0}, z, t_{0}\right)$, making the changes $E \rightarrow E^{\prime}$ and $t \rightarrow z$. From the definition of the operator $\hat{\Sigma}_{N}$, Eq. (9), it is easy to see that

$$
\hat{\Sigma}_{N} \delta\left(E^{\prime \prime}-E^{\prime}\right)=g\left(E^{\prime \prime}-E^{\prime}\right)
$$

So, it is left to us to identify the term in curly brackets on the right-hand side of Eq. (15). But it is exactly the expression of the formal solution for the nucleonic component, Eq. (12), provided we make the substitutions

$$
\begin{aligned}
E_{0} & \rightarrow E^{\prime \prime}, \\
T_{N} & \rightarrow \frac{t-z}{\lambda_{\pi}}, \\
\beta_{N} & \rightarrow \beta_{\pi} .
\end{aligned}
$$

Putting it all together and performing some integrations, we can finally write the explicit solution for the pionic component, in the energy configuration space, without any mathematical approximation:

$$
\begin{aligned}
F_{\pi}\left(E, E_{0}, t, t_{o}\right)= & \frac{\lambda_{\pi}}{\lambda_{\pi}-\lambda_{N}} g_{N}\left(E, E_{0}\right)\left[e^{-T_{\pi}}-e^{-T_{N}}\right] \\
& +\int_{t_{0}}^{t} \frac{d z}{\lambda_{N}} e^{-T(z)} \int_{E}^{E_{0}} \frac{d E^{\prime}}{E}\left(\frac{E}{E^{\prime}}\right)^{1+\beta_{\pi}}\left(1+\beta_{\pi}\right) \frac{t-z}{\lambda_{\pi}} \frac{2 I_{1}\left(u_{\pi}\right)}{u_{\pi}} g_{N}\left(E^{\prime}, E_{0}\right) \\
& +\int_{t_{0}}^{t} \frac{d z}{\lambda_{N}} e^{-T(z)} \int_{E}^{E_{0}} \frac{d E^{\prime}}{E}\left(\frac{E}{E_{0}}\right)^{1+\beta_{N}}\left(1+\beta_{N}\right) \frac{z-t_{0}}{\lambda_{N}} \frac{2 I_{1}\left(u_{N}\right)}{u_{N}} g_{N}\left(E^{\prime}, E_{0}\right) \\
& +\int_{t_{0}}^{t} \frac{d z}{\lambda_{N}} e^{-T(z)} \int_{E}^{E_{0}} \frac{d E^{\prime \prime}}{E^{\prime \prime}} \int_{E^{\prime \prime}}^{E_{0}} \frac{d E^{\prime}}{E}\left(\frac{E}{E^{\prime \prime}}\right)^{1+\beta_{\pi}}\left(\frac{E^{\prime \prime}}{E^{\prime}}\right)^{1+\beta_{N}} \\
& \times\left(1+\beta_{\pi}\right)\left(1+\beta_{N}\right) \frac{t-z}{\lambda_{\pi}} \frac{z-t_{0}}{\lambda_{N}} \frac{2 I_{1}\left(\overline{u_{\pi}}\right)}{u_{\pi}} \frac{2 I_{1}\left(\overline{u_{N}}\right)}{u_{N}} g_{N}\left(E^{\prime}, E_{0}\right),
\end{aligned}
$$

where we used

$$
\begin{aligned}
T_{\pi} & =\frac{t-t_{0}}{\lambda_{\pi}} \\
T(z) & =\frac{t-z}{\lambda_{\pi}}+\frac{z-t_{0}}{\lambda_{N}} \\
u_{N} & =2\left[\left(1+\beta_{N}\right) \frac{z-t_{0}}{\lambda_{N}} \ln \left(E^{\prime} / E\right)\right]^{1 / 2}, \\
u_{\pi} & =2\left[\left(1+\beta_{\pi}\right) \frac{(t-z)}{\lambda_{\pi}} \ln \left(E^{\prime} / E\right)\right]^{1 / 2}, \\
\overline{u_{N}} & =2\left[\left(1+\beta_{N}\right) \frac{\left(z-t_{0}\right)}{\lambda_{N}} \ln \left(E^{\prime} / E^{\prime \prime}\right)\right]^{1 / 2}, \\
\overline{u_{\pi}} & =2\left[\left(1+\beta_{\pi}\right) \frac{(t-z)}{\lambda_{\pi}} \ln \left(E^{\prime \prime} / E\right)\right]^{1 / 2} .
\end{aligned}
$$


The integral flux of hadrons is easily calculated from Eqs. (14) and (16):

$$
\left.I_{h}(\rangle E, E_{0}, t, t_{0}\right)=\int_{E}^{E_{0}} d E^{\prime}\left[F_{N}\left(E^{\prime}, E_{0}, t, t_{0}\right)+F_{\pi}\left(E^{\prime}, E_{0}, t, t_{0}\right)\right] .
$$

\section{COMPARISION WITH EXPERIMENTAL DATA}

\section{A. Multiparticle production with Feynman scaling}

Ohsawa and Yamashita [16] have proposed, in accordance with accelerator data on $p p \rightarrow \pi+X[20]$, an empirical expression for the production spectrum of charged pions in multiparticle production, respecting Feynman scaling [21]. It can be written as

$$
g_{N}\left(E, E_{0}\right) d E=\frac{5}{3}\left[1-\frac{E}{E_{0}}\right]^{4} \frac{d E}{E} .
$$

This expression leads to a constant mean inelasticity $\langle K\rangle=1 / 2$. Energy conservation imposes $\left\langle\sigma_{i}\right\rangle=1 / 2$ and, in this case, the simplest choice is the uniform distribution for the elasticities, $f\left(\sigma_{i}\right)=1$.

We compare now the integral energy spectrum of hadrons, calculated by Eq. (17), with the experimental data for the family Ursa Maior, as shown in Fig. 1. The spectrum is presented in fractional form, that is, as a function of the total hadronic visible energy:

$$
f_{h}=\frac{E_{h}^{\gamma}}{\sum E_{h}^{\gamma}}
$$

The hadronic visible energy $E_{h}^{\gamma}$ is the energy of hadrons detected by means of the electromagnetic showers induced by $\gamma$ rays from $\pi^{0}$ decay. It is related to the actual energy of the hadron $E_{h}$ by the so-called "gamma inelasticity" $k_{\gamma}$, so that

$$
E_{h}^{\gamma}=k_{\gamma} E_{h}
$$

We use here, for the gamma inelasticity, the mean value $\left\langle k_{\gamma}\right\rangle=0.25$.

The best fit calculation using Feynman scaling multiparticle production, Eq. (18), is indicated by the dotted curve in Fig. 1 (labeled FS). The free parameters were the primary nucleon visible energy $E_{0}^{\gamma}$ and the interaction height in the atmosphere $\left(t-t_{0}\right)$.

We see that it is not possible to describe these emulsion-chamber family data adopting the Feynman scaling law, at such high energies.

\section{B. Violation of Feynman scaling}

For the violation of Feynman scaling in multiparticle production we take the parametrization of Bellandi et al. [8]:

$$
g_{N}\left(E, E_{0}\right) d E=\frac{5}{3}\left(\frac{E_{0}}{E_{s}}\right)^{\alpha}\left[1-\left(\frac{E_{0}}{E_{s}^{\prime}}\right)^{\alpha^{\prime}} \frac{E}{E_{0}}\right]^{4} \frac{d E}{E} .
$$

The parameters used in Eq. (20) are those obtained by Ohsawa and Sawayanagi [9], adjusted to fit the pseudorapidity distribution $d N / d \eta$ data measured at the accelerator energies $\sqrt{s}=53,200,546,630$, and $900 \mathrm{GeV}$ :

$$
\begin{aligned}
& \alpha=0.11, \quad E_{S}=3.4 \times 10^{2} \mathrm{GeV}, \\
& \alpha^{\prime}=0.17, \quad E_{S}^{\prime}=9.6 \times 10^{2} \mathrm{GeV} .
\end{aligned}
$$

To compare the analytical result with experimental data we need to specify values for the parameters $\beta_{i}$ in the elasticity fluctuation distributions. We choose three different situations, combining nucleonic and pionic distributions.

Case (1) "Uniform:" $\beta_{N}=\beta_{\pi}=0$ so that $\left\langle\sigma_{N}\right\rangle=$

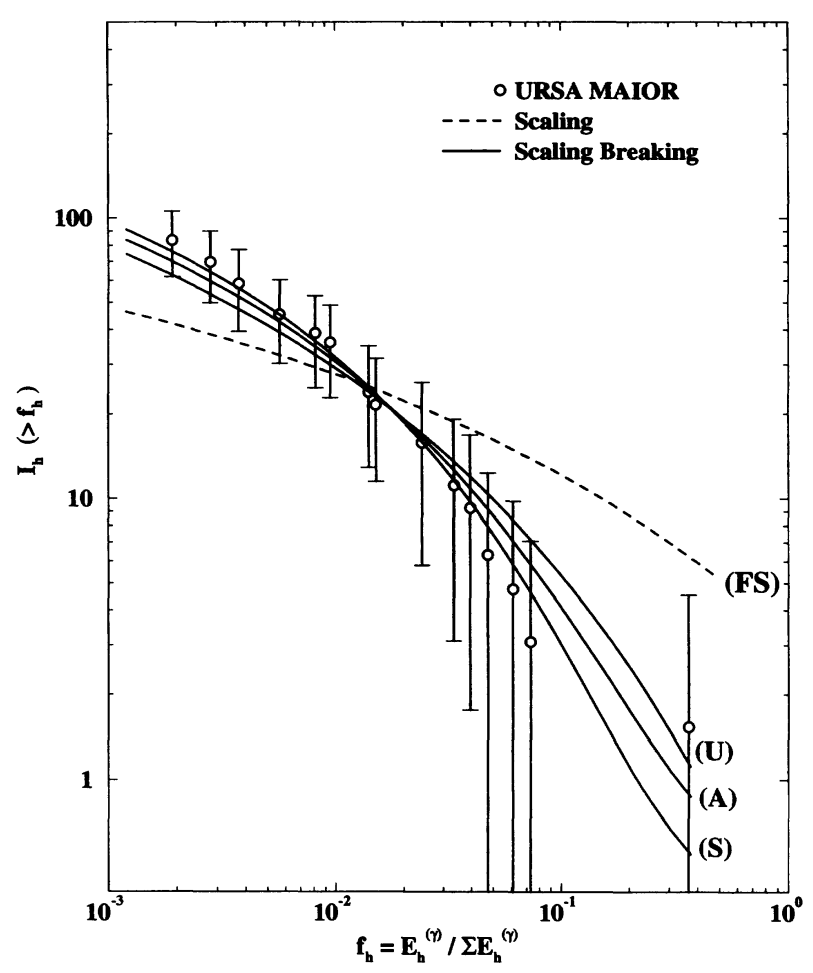

FIG. 1. Integral energy spectrum of hadrons in fractional form, for the cosmic-ray family Ursa Maior $\left(\Sigma E_{h}^{\gamma}=830 \mathrm{TeV}\right)$, detected at Mt. Chacaltaya $\left(t=540 \mathrm{~g} / \mathrm{cm}^{2}\right)$. Dotted curve (labeled FS) stands for the fit with Feynman scaling multiparticle production. Full curves are an analytical fit, with violation of the scaling law, for the three cases of elasticity distributions discussed in the text: $(U)=$ uniform, $(A)=$ asymmetric, $(S)=$ symmetric. 
$\left\langle\sigma_{\pi}\right\rangle=1 / 2$.

Case (2) "Asymmetric:" $\beta_{N}=\frac{1}{\left\langle K\left(E_{0}\right)\right\rangle}-2$ and $\beta_{\pi}=0$ so that $\left\langle\sigma_{N}\right\rangle=1-\left\langle K\left(E_{0}\right)\right\rangle$ and $\left\langle\sigma_{\pi}\right\rangle=1 / 2$.

Case (3) "Symmetric:" $\beta_{N}=\beta_{\pi}=\frac{1}{\left\langle K\left(E_{0}\right)\right\rangle}-2$ so that $\left\langle\sigma_{N}\right\rangle=\left\langle\sigma_{\pi}\right\rangle=1-\left\langle K\left(E_{0}\right)\right\rangle$, where the value of $\left\langle K\left(E_{0}\right)\right\rangle$ is calculated consistently with the multiparticle production spectra [8]:

$$
\left\langle K\left(E_{0}\right)\right\rangle=\frac{1}{2}\left(\frac{E_{0}}{E_{s}}\right)^{\alpha}\left(\frac{E_{0}}{E_{s^{\prime}}}\right)^{-\alpha^{\prime}} .
$$

We may now turn back to Fig. 1 and compare our calculations with the hadronic integral spectrum of Ursa Maior. There are three different full curves representing the best fit to experimental data, for each of the three cases of elasticity distribution: $(U)=$ uniform, $(A)=$ asymmetric, and $(S)=$ symmetric.

Again the adjusted parameters are the primary particle visible energy $E_{0}^{\gamma}$, which is related to the primary energy $E_{0}$ by Eq. (19), and the interaction height $\left(t-t_{0}\right)$, which describes the path developed by the cascade between the depth of primary interaction, $t_{0}$, and the detection level $t$ measured in units of $\mathrm{g} / \mathrm{cm}^{2}$. For Mt. Chacaltaya we have $t=540 \mathrm{~g} / \mathrm{cm}^{2}$. The results obtained by the fit for the different cases of elasticity distributions are presented in Table I.

We realize that the introduction of scaling breaking in multiparticle production makes it possible to achieve a very good description of the experimental data, for all combinations of elasticity distributions. Even so, little differences among $\chi^{2}$ values result from a $\chi^{2}$ minimization by using the CERN routine MINUIT (as indicated in Table I), showing a slight preference for the symmetric case. In fact, the assumption that $f\left(\sigma_{N}\right)=f\left(\sigma_{\pi}\right)$ is in accordance with a previous analysis [18] of Mt. Fuji Collaboration data. This case also leads to the lowest primary visible energy $E_{0}^{\gamma}=4920 \mathrm{TeV}$, which corresponds in the c.m. system (c.m.s.) to the primary particle energy of $\sqrt{s} \approx 6 \mathrm{TeV}$. In Table I we also see that all the fits to Ursa Maior data lead to the same value of interaction height, $\left(t-t_{0}\right)=540 \mathrm{~g} / \mathrm{cm}^{2}$, corresponding to the whole atmosphere above the emulsion chamber.

In Fig. 2 we show the results for the family P3'-C1B90, detected at Pamir $\left(t=596 \mathrm{~g} / \mathrm{cm}^{2}\right)$. Again, the symmetric case leads to a lower primary visible energy, $E_{0}^{\gamma}=1140 \mathrm{TeV}$ (see Table I), corresponding in the c.m.s. to the primary energy $\sqrt{s} \approx 3 \mathrm{TeV}$. The interaction

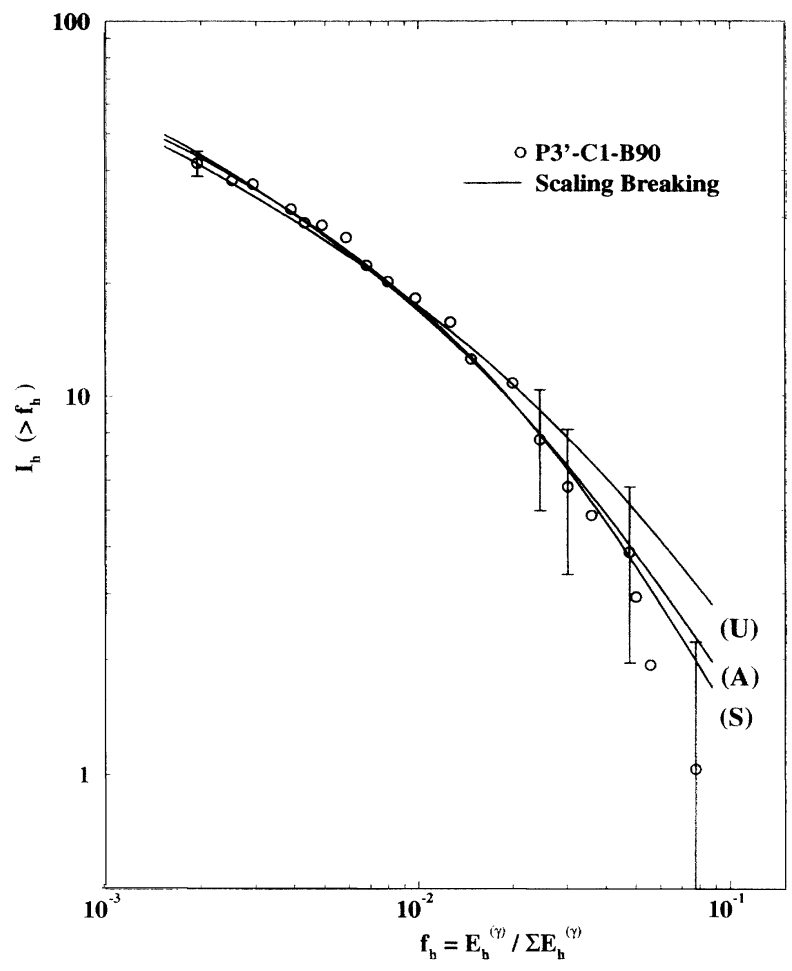

FIG. 2. Integral energy spectrum of hadrons for the cosmic-ray family P3'-C1-B90 $\left(\Sigma E_{h}^{\gamma}=230 \mathrm{TeV}\right)$, detected at Mt. Pamir $\left(t=596 \mathrm{~g} / \mathrm{cm}^{2}\right)$. Full curves are an analytical fit, with violation of the scaling law, for the three cases of elasticity distributions discussed in the text: $(U)=$ uniform, $(A)=$ asymmetric,$(S)=$ symmetric.

heights obtained by the fits range from $\left(t-t_{0}\right)=596$ $\mathrm{g} / \mathrm{cm}^{2}$ (the whole atmosphere above the detection level), to $\left(t-t_{0}\right)=402 \mathrm{~g} / \mathrm{cm}^{2}$ (for the symmetric case).

Although exceptional results obtained here for the emulsion-chamber superfamilies, with a total visible energy around $2000 \mathrm{TeV}$, exotic events are not well described on the same basis, even if their total energies are not very high. In Fig. 3 we show the experimental data of the event Centauro I, detected at Mt. Chacaltaya by the Brazil-Japan Collaboration, which has $\Sigma E_{\text {vis }}=330$ $\mathrm{TeV}$, compared to the tentative to fit data adopting violation of the scaling law, together with symmetric elasticity distributions.

TABLE I. Parameters obtained from the fits to integral energy spectrum of hadrons displayed in Figs. 1, 2, and 3.

\begin{tabular}{ccccccc}
\hline \hline Event & $f(\sigma)$ & $E_{0}^{\gamma}(\mathrm{TeV})$ & $t-t_{0}\left(\mathrm{~g} / \mathrm{cm}^{2}\right)$ & $\beta_{N}$ & $\beta_{\pi}$ & $\chi^{2}$ \\
\hline \multirow{3}{*}{ Ursa Maior } & Scaling & 84200 & 540 & 0 & 0 & 82.4 \\
& Uniform & 23000 & 540 & 0 & 0 & 4.8 \\
& Asymmetric & 8680 & 540 & 1.35 & 0 & 2.0 \\
& Symmetric & 4920 & 540 & 1.23 & 1.23 & 0.6 \\
\hline \multirow{3}{*}{ P3'-C1-B90 } & Uniform & 6730 & 596 & 0 & 0 & 13.3 \\
& Asymmetric & 2340 & 568 & 1.09 & 0 & 5.3 \\
& Symmetric & 1140 & 402 & 0.96 & 0.96 & 3.4 \\
\hline Centauro I & Symmetric & 2110 & 540 & 1.07 & 1.07 & 887.5 \\
\hline \hline
\end{tabular}




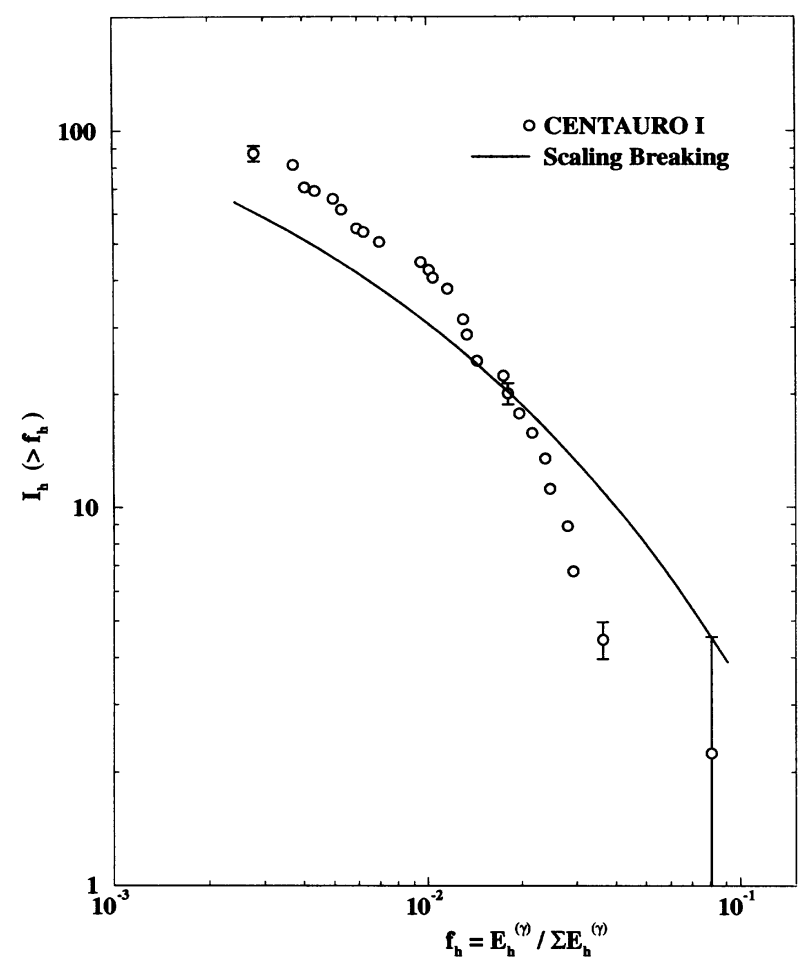

FIG. 3. Integral energy spectrum of hadrons for the cosmic-ray event Centauro I $\left(\Sigma E_{h}^{\gamma}=330 \mathrm{TeV}\right)$, detected at Mt. Chacaltaya. The full curve is the analytical fit, with violation of the scaling law, for the symmetric case of elasticity distributions.

\section{DISCUSSION}

We have obtained analytical solutions for the diffusion equations of the hadronic cascade induced by one single nucleon in the atmosphere. With these solutions we calculated the hadronic integral spectrum in order to describe emulsion-chamber experimental data from the superfamilies Ursa Maior (Mt. Chacaltaya) and P3'-C1B90 (Mt. Pamir).

We have also shown to what extent Feynman scaling combined with our cascading formalism fails to describe these experimental data (curve FS in Fig. 1). It was also demonstrated that an excellent agreement between those cosmic-ray data and our analytical calculation can be achieved incorporating scaling breaking in the rapidity density distributions. So, it becomes clear from this analysis that our formalism requires Feynman scaling breaking to describe ultrahigh energy events initiated by one single nucleon in the atmosphere.

The multiparticle production model used here implies a smooth decrease of mean inelasticity with energy. It must be emphasized that in this model the parametrization for rapidity distributions was obtained to fit data from the CERN Intersecting Storage Rings (ISR) and Super Proton Synchrotron (SPS), remaining consistent with data up to Fermilab Tevatron energies. However, the behavior of the mean inelasticity at superigh energies is a rather controversial subject and it is beyond the scope of this work to face this question in a detailed way (about this problem, see Ref. [22] and references quoted therein). In the present case, the reason for obtaining such agreement between our theoretical results and the experimental superfamily data is probably due to the enhancement of the leading particle effect, implied by the rapidity distributions borrowed from Ref. [9]. In fact, it can be seen from Fig. 1, Fig. 2, and Fig. 3 that the experimental point with the highest $f_{h}$ falls apart from the others in the energy spectrum, indicating the existence of a strong leading particle effect. This implies a large mean elasticity for the events. Our calculations reflect this fact in the values obtained for $\beta$. From Table $I$, we see that the values lie near $\langle\beta\rangle \approx 1$, in the symmetric case $\left[f\left(\sigma_{N}\right)=f\left(\sigma_{\pi}\right)\right]$, which is the most favorable one. According to Eq. (5) this corresponds to a linear growing distribution of elasticity, $f(\sigma) \approx 2 \sigma$, favoring collisions with high elasticities. Actually, from an experimental analysis of isodensitometric maps for huge halo events [3], it is concluded that these shower clusters are strongly penetrative, indicating a dominant leading particle effect.

Finally, we mention that other high energy cosmicray families were tested and similar results were found provided the total visible energy of the event is around $2000 \mathrm{TeV}$ so that the parametrization for the multiparticle production spectra remains within its validity range. However, we see that tentatives to reproduce exotic events, such as Centauro I, under the same basis stated above, were not successful. The reason is that those exotic events consist mostly of baryons, with a still unexplained lack of $\gamma$ rays, and a more complex treatment has to be considered [23]. Remembering that the most energetic experimental point in the integral spectrum of Centauro I has a very large error bar, centered near $I_{h}=2$ (see Fig. 3), we suggest the possibility that there are two energetic nucleons in the development of the cascade which could be treated analytically by introducing the production of nucleons by nucleons. Another way of facing this problem should be to consider Centauro-like events as a result of the interaction of heavier primaries with the atmosphere. In fact, the study of the chemical composition of the primary cosmic radiation around and above the "knee" of the spectrum $(\geq 3000 \mathrm{TeV})$ suggests a mixed composition with higher fraction of heavy nuclei than at energies below this region [15]. In any case, both possibilities would require the development of new formalisms, modifying our premises in a substantial way. In order to improve our knowledge and understanding about this subject, there is also the need of more data to be analyzed, mainly in the fragmentation region.

\section{ACKNOWLEDGMENTS}

We thank Professor Akinori Ohsawa (University of Tokyo) for useful comments on the multiparticle production model. This work was partially supported by FAPESP and CNPq (Brazil). 
[1] C.G.M. Lattes, Y. Fujimoto, and S. Hasegawa, Phys. Rep. 65, 151 (1980).

[2] Pamir Collaboration, Mt. Fuji Collaboration and Chacaltaya Collaboration, S.G. Bayburina et al., Nucl. Phys. B191, 1 (1981).

[3] Chacaltaya and Pamir Collaboration, L.T. Baradzei et al. Nucl. Phys. B370, 365 (1992).

[4] S. Yamashita, J. Phys. Soc. Jpn. 54, 529 (1985).

[5] Brazil-Japan Collaboration, J.A. Chinellato et al., in Proceedings of the XXI International Cosmic Ray Conference, Adelaide, Australia, edited by R.J. Protheroe (Graphic Services, Northfield, South Australia, 1990), Vol. 8, p. 259.

[6] Brazil Group, C.G.M. Lattes et al. and Japan group, M. Akashi et al., Prog. Theor. Phys. Suppl. 47, 1 (1971).

[7] J.A. Chinellato, Ph.D. thesis, Universidade Estadual de Campinas, 1981.

[8] J. Bellandi Fo , S.Q. Brunetto, J.A. Chinellato, C. Dobrigkeit, A. Ohsawa, K. Sawayanagi, and E.H. Shibuya, Prog. Theor. Phys. 83, 58 (1990).

[9] A. Ohsawa and K. Sawayanagi, Phys. Rev. D 45, 3128 (1992).

[10] UA5 Collaboration, G.J. Alner et al., Z. Phys. C 33, 1 (1986).

[11] UA7 Collaboration, E. Pare et al., Phys. Lett. B 242,
531 (1990).

[12] F. Abe et al., Phys. Rev. D 41, 2330 (1990).

[13] J. Wdowczyk and A. Wolfendale, Nuovo Cimento A 54, 433 (1979); J. Phys. G 10, 257 (1984); 13, 411 (1987).

[14] J. Bellandi, L.M. Mundim, J. Dias de Deus, and R.J.M. Covolan, J. Phys. G 18, 579 (1992).

[15] T.K. Gaisser et al., Phys. Rev. D 47, 1919 (1993).

[16] A. Ohsawa and S. Yamashita, Prog. Theor. Phys. 77, 1411 (1987).

[17] R. P. Feynman, Phys. Rev. 84, 108 (1951).

[18] J. Bellandi Fo , S.Q. Brunetto, J.A. Chinellato, R.J.M. Covolan, C. Dobrigkeit, and M.A. Alves, Nuovo Cimento C 14, 15 (1991).

[19] J. Bellandi, R.J.M. Covolan, C. Dobrigkeit, C.G.S. Costa, and L.M. Mundim, J. Phys. G 18, 1559 (1992).

[20] J.R. Johnson, R. Kammerud, T. Ohsugi, D.J. Ritchie, R. Shafer, D. Theriot, J.K. Walker, and F.E. Taylor, Phys. Rev. D 17, 1292 (1978).

[21] R.P. Feynman, Phys. Rev. Lett. 23, 1415 (1969).

[22] J. Bellandi, R.J.M. Covolan, C.G.S. Costa, J. Montanha, and L.M. Mundim, Phys. Rev. D 50, 297 (1994).

[23] See, for example, J.D. Bjorken and L.D. McLerran, Phys. Rev. D 20, 2353 (1979); A.D. Panagiotou, A. Petridis, and M. Vassiliou, ibid. D 45, 3134 (1992); R. Attallah and J.N. Capdevielle, J. Phys. G 19, 1381 (1993). 